\title{
Comportement rhéologique dans des écoulements transitoires en cisaillement simple des polymères fortement chargés en fibres courtes
}

\author{
Maryam Sepehr $^{1}$, Gilles Ausias ${ }^{2, a}$ et Pierre J. Carreau ${ }^{1}$ \\ ${ }^{1}$ CRASP, Département de Génie Chimique, École polytechnique de Montréal, C.P. 6079, Succ. Centre-Ville, Montréal, \\ QC, H3C 3A7, Canada \\ 2 LG2M, Université de Bretagne Sud, Rue de St Maudé, BP 92116, 56321 Lorient Cedex, France
}

Reçu le 10 juin 2003, accepté le 7 novembre 2003

\begin{abstract}
Résumé - Le comportement d'un polypropylène chargé de fibres de verre courtes a été étudié. À l'état fondu, un thermoplastique renforcé de fibres courtes ayant un état d'orientation initial isotrope présente un pic de viscosité lorsqu'il est cisaillé entre les deux disques d'un rhéomètre à géométrie plan-plan. Dans cette étude, après une première déformation, l'échantillon a été cisaillé dans la direction inverse et un pic de viscosité a été à nouveau mesuré. De même des pics de contraintes normales ont été observés. Dans l'écoulement inverse les contraintes normales passent par des valeurs négatives puis présentent un pic positif. Un modèle a été utilisé pour simuler ces pics de viscosité et de contraintes normales. Il est constitué de l'équation de Folgar-Tucker pour le mouvement des fibres et de l'équation de Lipscomb pour la loi de comportement. Ces équations ont été établies pour des solutions diluées ou semi-diluées de fibres. Ces composites industriels sont considérés comme des suspensions concentrées, les fibres sont proches de leurs voisines et leurs rotations sont plus lentes que ce que prédit la simulation. Ce modèle a été empiriquement modifié pour simuler correctement les pics lorsque le taux de fibre augmente.
\end{abstract}

Mots clés : Rhéologie / polymère chargé / fibre courte / mise en forme des polymères / modèle / orientation / solution concentrée

\begin{abstract}
Rheological behavior in transient shear flows for concentrated short fiber filled polymer. The behaviour of commercial short glass fibre filled polypropylene behaviour has been studied. A molten thermoplastic filled polymer containing short fibres with an initial isotropic orientation state exhibits a viscosity overshoot when sheared in parallel plate geometry of a rheometer. In this study, after a first deformation, the sample has been sheared in the reverse direction and an other viscosity overshoot has been observed. Similarly, normal stress overshoots were observed. In the reverse flow the normal stresses were initially negative and then exhibited a positive overshoot. A model has been used to describe these viscosity and normal stress overshoots. It is based on the Folgar-Tucker equation for fibre motion and the Lipscomb constitutive equation. These equations have been established for dilute or semi-dilute solutions. These industrial composites are considered as concentrated suspensions; the fibres are close to their neighbours and fibre rotation is slower than predicted by the model. This model has been empirically modified to correctly describe the overshoots with increasing fibre content.
\end{abstract}

Key words: Rheology / filled polymer / short fibre / polymer processing / model / orientation / concentrated solution

\section{Introduction}

Les thermoplastiques sont des polymères présentant de nombreux avantages (prix, facilité de mise en œuvre, légèreté, complexité des formes obtenues, absence

\footnotetext{
a Auteur correspondant : gilles.ausias@univ-ubs.fr
}

de corrosion, possibilité de recyclage...) et sont de plus en plus utilisés dans l'automobile, l'aéronautique, l'électroménager. Cependant, pour palier la faiblesse de leurs propriétés mécaniques qui constituent un frein à leur utilisation, ils sont très souvent renforcés par des fibres courtes. Le comportement d'une pièce fabriquée avec ce type de composite (thermoplastique + fibres) dépendra de 


\section{Nomenclature}

\begin{tabular}{|c|c|c|}
\hline $\mathbf{a}_{2}$ & tenseur d'orientation d'ordre 2 & \\
\hline$\dot{\mathbf{a}}_{2}$ & dérivée particulaire du tenseur d'orientation d'ordre 2 & \\
\hline $\mathbf{a}_{4}$ & tenseur d'orientation d'ordre 4 & \\
\hline $\mathbf{a}_{4}^{l}, \mathbf{a}_{4}^{q}, \mathbf{a}_{4}^{h}$ & tenseur d'orientation d'ordre 4 estimé à l'aide des approximations & \\
\hline & linéaire, quadratique et hybride & \\
\hline$a_{1}, b_{1}$ & coefficients du calcul de la distance moyenne entre 2 fibres & \\
\hline$C_{\gamma}^{\exp }$ & couple mesuré à un niveau de déformation $\gamma$ & N.m \\
\hline$C_{\gamma}^{\mathrm{cal}}$ & couple calculé à un niveau de déformation $\gamma$ & N.m \\
\hline$C_{\gamma_{\infty}}^{\exp }$ & couple mesuré à un niveau de déformation infini & N.m \\
\hline$C_{I}$ & coefficient de diffusion & \\
\hline$C_{I}^{*}$ & coefficient de diffusion & \\
\hline$f, g$ & fonctions d'orientation & \\
\hline$H$ & distance moyenne entre 2 fibres & \\
\hline $\mathbf{I}$ & tenseur unité & \\
\hline$P$ & pression hydrostatique & $\mathrm{Pa}$ \\
\hline $\mathbf{p}$ & vecteur unitaire parallèle à l'axe de révolution de la fibre & \\
\hline$r$ & rapport de forme des fibres & \\
\hline$R$ & rayon des plateaux du rhéomètre & $\mathrm{m}$ \\
\hline$\alpha$ & coefficient de glissement & \\
\hline$\phi$ & fraction volumique de fibre & \\
\hline$\phi_{0}$ & fraction volumique d'une fibre & \\
\hline$\dot{\gamma}$ & tenseur des vitesses de déformation & $\mathrm{s}^{-1}$ \\
\hline$\dot{\bar{\gamma}}$ & taux de cisaillement généralisé & $\mathrm{s}^{-1}$ \\
\hline$\eta_{0}$ & viscosité dynamique de la matrice & Pa.s \\
\hline$\eta_{\gamma}^{\exp }$ & viscosité expérimentale à un niveau de déformation $\gamma$ & Pa.s \\
\hline$\eta_{\gamma_{\infty}}^{\exp }$ & viscosité expérimentale à un niveau de déformation infini & Pa.s \\
\hline$\eta_{r}^{\exp }$ & viscosité relative expérimentale & \\
\hline$\kappa$ & tenseur des gradients de vitesse & $\mathrm{s}^{-1}$ \\
\hline$\lambda$ & coefficient de forme des fibres & \\
\hline$\mu_{0}, \mu_{1}, \mu_{2}, \mu_{3}$ & coefficients rhéologiques du modèle de Lipscomb & \\
\hline$\sigma$ & tenseur des contraintes dans le composite & $\mathrm{Pa}$ \\
\hline$\sigma_{f}$ & contribution du fluide (la matrice) au tenseur des contraintes & $\mathrm{Pa}$ \\
\hline$\sigma_{s}$ & contribution des particules (les fibres) au tenseur des contraintes & $\mathrm{Pa}$ \\
\hline$\sigma_{p}$ & contribution d'une particule (la fibre) au tenseur des contraintes & $\mathrm{Pa}$ \\
\hline$\psi(\mathbf{p})$ & fonction de distribution d'orientation des fibres & \\
\hline$\Omega$ & tenseur des vitesses de rotation & $\mathrm{s}^{-1}$ \\
\hline
\end{tabular}

l'orientation des fibres. Cette orientation est induite par l'écoulement du composite dans l'outil de mise en forme. Pour comprendre l'écoulement de ces matériaux et l'orientation des fibres pendant le procédé de mise en forme, il est nécessaire d'étudier leur comportement rhéologique à l'état fondu et d'établir des modèles permettant de le décrire correctement. Ces modèles servent à simuler les procédés de mise en forme, à prédire l'état d'orientation des fibres dans la pièce et éventuellement à modifier les outillages pour optimiser ces orientations. Un modèle et des expériences de caractérisation seront présentés. Ensuite une amélioration du modèle sera proposée. Enfin sera présenté un exemple de la manière dont on peut utiliser ces outils pour modéliser les procédés de mise en forme et un exemple permettant de comprendre comment ils peuvent être améliorés.

\section{Modèle}

Trois régimes de concentration de fibres peuvent être distingués. Le régime dilué où la quantité de fibres dans le composite est faible. Elles sont loin les unes des autres 
sans interagir entre elles. Le régime concentré où la quantité de fibres est importante comme c'est le cas des composites industriels. Il y a de nombreux contacts et interactions hydrodynamiques entre celles-ci. Des agglomérats de fibres peuvent être présents. Le troisième régime, intermédiaire, appelé semi-dilué est caractérisé par des interactions occasionnelles. La limite inférieure de ce régime est telle que $\phi=1 / r^{2}$ et la limite supérieure est telle que $\phi=1 / r$, où $\phi$ est la fraction volumique de fibres et $r$ le rapport de forme des fibres.

\subsection{Orientation des fibres}

L'orientation d'une fibre peut être décrite à l'aide d'un vecteur unitaire $\mathbf{p}$ parallèle à son axe de révolution. Pour une population de fibres en un point donné du composite, la fonction de distribution d'orientation $\psi(\mathbf{p})$ peut être utilisée pour décrire l'état d'orientation et les tenseurs d'orientation d'ordre 2 et d'ordre 4 sont définis par :

$$
\begin{aligned}
& \mathbf{a}_{2} \Leftrightarrow a_{i j}=\int_{\mathbf{p}} p_{i} p_{j} \psi(\mathbf{p}) \mathrm{d} \mathbf{p} \\
& \mathbf{a}_{4} \Leftrightarrow a_{i j k l}=\int_{\mathbf{p}} p_{i} p_{j} p_{k} p_{l} \psi(\mathbf{p}) \mathrm{d} \mathbf{p}
\end{aligned}
$$

Un bilan du moment sur le centre de gravité de la fibre nous conduit à une équation du mouvement en rotation du vecteur p (le modèle de Jeffery [1]). Pour une efficacité dans les calculs, une représentation compacte de cette équation a été suggérée avec une approche tensorielle. Dans cette méthode, le tenseur d'orientation d'ordre 4 apparaît. Généralement on utilise le tenseur d'ordre 2 pour représenter l'état d'orientation le tenseur d'ordre 4 étant inconnu. C'est pourquoi, ce dernier doit être évalué en utilisant une approximation de fermeture. Il existe les approximations linéaire $\mathbf{a}_{4}^{l}$, quadratique $\mathbf{a}_{4}^{q}$ ou hybride $\mathbf{a}_{4}^{h}$ calculées à partir de $\mathbf{a}_{2}$ et définies par Advani et Tucker [2] :

$$
\begin{aligned}
\mathbf{a}_{4}^{h} & =a_{i j k l}^{h}=(1-f) \mathbf{a}_{4}^{l}+f \mathbf{a}_{4}^{q} \\
\mathbf{a}_{4}^{l} & =a_{i j k l}^{l}=-\frac{1}{35}\left(\delta_{i j} \delta_{k l}+\delta_{i k} \delta_{j l}+\delta_{i l} \delta_{j k}\right) \\
& +\frac{1}{7}\left(a_{i j} \delta_{k l}+a_{i k} \delta_{j l}+a_{i l} \delta_{j k}+a_{k l} \delta_{i j}+a_{j l} \delta_{i k}+a_{j k} \delta_{i l}\right)
\end{aligned}
$$

$\mathbf{a}_{4}^{q}=a_{i j k l}^{q}=a_{i j} a_{k l}$

avec $f=\frac{1}{2}\left(-7-27 a_{i j} a_{j i}+18 a_{i j} a_{j k} a_{k i}\right)$.

Les deux familles d'approximations de fermeture les plus récentes sont les approximations orthotropiques (Cintra et Tucker [3] et Wetzel et Tucker [4]) et l'approximation naturelle (Verleye et Dupret [5]). Elles procèdent d'une même démarche. Les invariants de $\mathbf{a}_{2}$ sont utilisés pour calculer $\mathbf{a}_{4}$. Les axes principaux de $\mathbf{a}_{4}$ sont les mêmes que ceux de $\mathbf{a}_{2}$. Quand $\mathbf{a}_{4}$ est exprimé dans le repère de ces axes principaux, un certain nombre de ces composantes sont nulles tandis que les autres peuvent être exprimées en fonction des valeurs propres de $\mathbf{a}_{2}$.
Tableau 1. Correspondance entre les indices de $A_{m n}$ et de $a_{i j k l}$.

\begin{tabular}{cc}
\hline$m$ ou $n$ & $i j$ ou $k l$ \\
\hline 1 & 11 \\
2 & 22 \\
3 & 33 \\
4 & 23 ou 32 \\
5 & 31 ou 13 \\
6 & 12 ou 21 \\
\hline
\end{tabular}

Sa trace étant unitaire, seules deux valeurs propres $\lambda_{1}$ et $\lambda_{2}$, sont indépendantes. Utilisant certaines propriétés de $\mathbf{a}_{4}$, Cintra et Tucker [3] proposent d'utiliser une notation contractée : $A_{m n} \Leftrightarrow a_{i j k l}$. Chaque paire d'indices est remplacée par un indice unique allant de 1 à 6 suivant le tableau 1.

En utilisant d'autres propriétés de $\mathbf{a}_{4}$, il peut être décrit à l'aide de trois termes indépendants $\bar{A}_{11}, \bar{A}_{22}$ et $\bar{A}_{33}$ dans le repère des axes principaux. Ils sont ensuite écrits comme des fonctions polynomiales de $\lambda_{1}$ et $\lambda_{2}$. Différentes stratégies ont été utilisées pour déterminer les coefficients de ces polynômes [6]. Dans cette étude l'approximation proposée par Wetzel et Tucker [7] est utilisée.

\subsection{Mouvement des fibres}

Pour pouvoir comprendre comment les fibres s'orientent dans le procédé de mise en forme et savoir quelle sera l'orientation des fibres dans la pièce fabriquée, il est nécessaire de connaître leur mouvement. Dans l'écoulement, les fibres vont se déplacer et tourner sous l'effet des gradients de vitesse. Jeffery [1] établit les équations décrivant le mouvement d'une particule ellipsoïdale isolée dans un fluide newtonien déformé par un gradient de vitesse uniforme dans la cellule contenant la particule. Cette équation a donc été établie pour une solution diluée de fibres. Folgar et Tucker [8] ajoutent un terme de diffusion pour tenir compte des interactions entre les fibres dans une solution semi-diluée et obtiennent finalement :

$$
\begin{array}{r}
\dot{\mathbf{a}}_{2}=\frac{\mathrm{D} \mathbf{a}_{2}}{\mathrm{D} t}=\mathbf{\Omega} \cdot \mathbf{a}_{2}-\mathbf{a}_{2} \cdot \boldsymbol{\Omega}+\lambda\left(\dot{\gamma} \cdot \mathbf{a}_{2}+\mathbf{a}_{2} \cdot \dot{\gamma}-2 \dot{\gamma}: \mathbf{a}_{4}\right) \\
+2 C_{I} \dot{\bar{\gamma}}\left(\mathbf{I}-3 \mathbf{a}_{2}\right)
\end{array}
$$

où $\mathrm{D} / \mathrm{D} t$ est la dérivée particulaire, $\lambda=\left(r^{2}-1\right) /\left(r^{2}+1\right)$. $\dot{\gamma}$ et $\Omega$ sont les tenseurs des vitesses de déformation et de rotation définis respectivement par $\dot{\gamma}=1 / 2\left(\boldsymbol{\kappa}+\boldsymbol{\kappa}^{t}\right)$ et $\boldsymbol{\Omega}=1 / 2\left(\boldsymbol{\kappa}-\boldsymbol{\kappa}^{t}\right)$ où $\boldsymbol{\kappa}$ est le tenseur des gradients de vitesse. Le dernier terme de l'équation (6) est proportionnel au coefficient de diffusion $C_{I}$ et au taux de cisaillement généralisé défini par $\dot{\bar{\gamma}}=\sqrt{2(\dot{\gamma}: \dot{\gamma})}$. À partir d'un grand nombre d'expériences, d'observations et de simulations numériques sur des plaques injectées en 
thermoplastique, Bay [9] propose pour le coefficient de diffusion $C_{I}$ le modèle empirique suivant :

$$
C_{I}=0,0184 \exp (-0,7148 \phi r)
$$

Fan et al. [10] proposent un terme de diffusion qui ne soit pas isotrope, car les phénomènes d'interactions ne sont pas les mêmes suivant le plan dans lequel on se situe par rapport au plan de cisaillement. Le coefficient de diffusion n'est alors plus un scalaire, mais un tenseur. Phan-Thien et al. [11] ont déterminé une équation empirique pour le coefficient de diffusion en fonction du taux de fibres et de leur rapport de forme :

$$
C_{I}=A[1,0-\exp (-B \phi r)]
$$

où $A$ et $B$ sont deux paramètres qu'ils déterminent à partir de résultats d'expériences numériques réalisées sur l'écoulement de systèmes ayant différents taux de fibres et différents rapports de forme en cisaillement simple. Ils obtiennent $A=0,03$ et $B=0,224$. Ranganathan et Advani [12] ont évalué la distance moyenne entre fibres en fonction de l'état d'orientation et ont proposé un coefficient de diffusion proportionnel à cette distance moyenne entre fibres :

$$
C_{I}=\frac{C_{I}^{*}}{(H / l)}
$$

où $C_{I}^{*}$ est un coefficient de diffusion et $H$ la distance moyenne entre deux fibres calculée en fonction de la géométrie et de l'état d'orientation des fibres. Aït-Kadi et Grmela [13] évaluent cette distance moyenne à partir du coefficient $g$ tel que :

$$
\begin{aligned}
H & =a_{1}+b_{1} g \\
g & =1-\left(27 \operatorname{det} \mathbf{a}_{2}\right)^{0,2}
\end{aligned}
$$

avec

$$
\begin{aligned}
& a_{1}=\pi \frac{D^{2}}{4 \phi l} \\
& b_{1}=\sqrt{\pi \frac{D^{2}}{4 \phi}}-a_{1}
\end{aligned}
$$

\subsection{Loi de comportement}

Batchelor [14] propose d'introduire la contribution de chaque particule aux contraintes dans le fluide en ajoutant à la contrainte due à la viscosité du fluide $\boldsymbol{\sigma}_{f}$, la contrainte supplémentaire due aux particules $\boldsymbol{\sigma}_{s}$ sous la forme suivante :

$$
\boldsymbol{\sigma}=\boldsymbol{\sigma}_{f}+\boldsymbol{\sigma}_{s}=-P \mathbf{I}+2 \eta_{0} \dot{\gamma}+\phi_{0} \sum \boldsymbol{\sigma}_{p}
$$

où $P$ est la pression hydrostatique, I est le tenseur unité, $\eta_{0}$ est la viscosité de la matrice supposée newtonienne, $\phi_{0}$ est la fraction volumique d'une particule dans le volume élémentaire choisi et $\boldsymbol{\sigma}_{p}$ le tenseur des contraintes induites par la présence de la particule. Pour des particules sphériques la contribution est isotrope et intervient uniquement sous la forme d'une augmentation de viscosité. Pour une suspension diluée de sphères, Einstein établit une équation qui peut se mettre sous la forme :

$$
\boldsymbol{\sigma}=-P \mathbf{I}+2 \eta_{0} \dot{\gamma}(1+2,5 \phi)
$$

C'est l'équation constitutive la plus simple et la plus connue pour un système chargé de particules. Elle s'applique aux charges sphériques en solution diluée, et le comportement du système reste isotrope. Pour les particules non-sphériques, il est intéressant de prendre comme point de départ les travaux d'Ericksen [15]. À partir de considérations physiques et mathématiques, il établit une théorie sur les fluides anisotropes transverses (Transversaly Isotropic Fluid) dont l'anisotropie est définie en tout point par un vecteur directeur p. Il ne garde que les termes linéaires en gradients de vitesse et obtient l'équation :

$\boldsymbol{\sigma}=-P \mathbf{I}+\mu_{0} \dot{\gamma}+\left(\mu_{1}+\mu_{2} \dot{\gamma}: \mathbf{p p}\right) \mathbf{p p}+2 \mu_{3}(\dot{\gamma} \cdot \mathbf{p p}+\mathbf{p p} \cdot \dot{\gamma})$

où les coefficients $\mu_{i}$ sont des coefficients rhéologiques et pp le produit du vecteur $\mathbf{p}$ par lui-même est un tenseur d'ordre deux. Lipscomb [16] reprend cette équation et à l'aide de la fonction de distribution d'orientation $\psi(\mathbf{p})$ la réécrit en fonction des tenseurs d'orientation. Il propose la forme générale que doit prendre l'équation constitutive pour une suspension de particules et dont l'état d'orientation de la structure est décrit à l'aide des tenseurs d'orientation :

$$
\begin{aligned}
\boldsymbol{\sigma}=-P \mathbf{I}+ & 2 \eta_{0} \dot{\gamma}+\eta_{0} \phi\left[P_{0} \mathbf{I}+\mu_{0} \dot{\gamma}\right. \\
& \left.+\mu_{1} \mathbf{a}_{2}+\mu_{2} \dot{\gamma}: \mathbf{a}_{4}+2 \mu_{3}\left(\dot{\gamma} \cdot \mathbf{a}_{2}+\mathbf{a}_{2} \cdot \dot{\gamma}\right)\right]
\end{aligned}
$$

où $P_{0}$ est la contribution des particules à la pression hydrostatique. Il propose également une évaluation des coefficients $\mu_{i}$. Il s'appuie sur les travaux de Hand [17] et Giesekus [18] et propose une valeur aux coefficients $\mu_{i}$. Il obtient finalement pour des fibres de grands rapports de forme :

$$
\boldsymbol{\sigma}=-P \mathbf{I}+2 \eta_{0} \dot{\gamma}+\eta_{0} \phi\left(4 \dot{\gamma}+\mu_{2} \dot{\gamma}: \mathbf{a}_{4}\right)
$$

avec $\mu_{2}$, le coefficient de couplage :

$$
\mu_{2}=\frac{r^{2}}{\ln r}
$$

Le modèle, formé de deux équations (6) et (15) couplées qui dépendent toutes les deux de la valeur actuelle de l'orientation et des gradients de vitesse, peut être présenté sous la forme :

$$
\left\{\begin{array}{l}
\boldsymbol{\sigma}=h^{\prime}\left(\boldsymbol{\kappa}, \mathbf{a}_{2}, \mathbf{a}_{4}\right) \\
\dot{\mathbf{a}}_{2}=h^{\prime \prime}\left(\boldsymbol{\kappa}, \mathbf{a}_{2}, \mathbf{a}_{4}\right)
\end{array}\right.
$$

Il faut ajouter à ces deux équations une approximation de fermeture. 


\section{4 Écoulement sous cisaillement simple}

Le modèle constitué des équations (6) et (15) et d'une approximation de fermeture a été utilisé pour simuler le comportement transitoire d'un polymère chargé de fibres courtes en cisaillement simple pour des écoulements « aller $»$ et « retour $»$. La déformation $\gamma$ est égale au produit du taux de cisaillement $\dot{\gamma}$ par le temps $t$. En utilisant l'équation (15), la viscosité est égale à :

$$
\eta=\frac{\sigma_{12}}{\dot{\gamma}}=2 \eta_{0}\left[1+\phi\left(2+\mu_{2} a_{1212}\right)\right]
$$

Dans ce calcul, les différents paramètres sont fixés aux valeurs suivantes : $\eta_{0}=1$ Pa.s, $C_{I}=0,01, r=20, \phi=0,1$ et $\mu_{2}=100$. L'orientation initiale des fibres est isotrope.

Dans des écoulements transitoires en cisaillement simple, on constate l'apparition d'un pic de viscosité au démarrage, déjà observé dans des expériences et dans des simulations avec ce modèle (Ausias et al. [19]). Ensuite la viscosité atteint le plateau du régime permanent (Fig. 1). En changeant immédiatement le sens du cisaillement pour un échantillon cisaillé, un pic de viscosité réapparaît avec une plus faible amplitude à une plus grande déformation.

Les différents types d'approximations de fermeture ont été vérifiés. La forme du pic dépendra de l'approximation choisie. On observe que le calcul avec l'approximation linéaire produit des oscillations. On remarque que les allures du pic aller avec l'approximation hybride et du pic retour avec l'approximation quadratique ne sont pas conformes à celles observées dans les expériences (Sect. 3). Aussi, pour une meilleure corrélation avec l'expérience, l'approximation de fermeture orthotrope proposée par Wetzel et Tucker [7] a été choisie.

L'influence du coefficient $C_{I}$ a été également étudiée. Lorsqu'il est égal à zéro, le terme de diffusion est nul. Seul les termes de convection de Jeffery [1] (Éq. (6)) restent et les évolutions de l'orientation et de la viscosité sont réversibles (Fig. 2). Pour des valeurs égales à 0,02 ou 0,002 , un pic à l'aller et un pic au retour ont été obtenus. La largeur du pic à l'aller reste de l'ordre de 10 unités de déformation et n'est pratiquement pas modifiée par la valeur de ce coefficient. Par contre, il modifie fortement l'allure du pic retour (Fig. 2).

Ces simulations permettent de calculer l'état d'orientation des fibres. L'état d'orientation initial est isotrope. La durée du pic de viscosité correspond au temps nécessaire aux fibres pour basculer et s'orienter dans le sens de l'écoulement. Au début de l'écoulement retour, cette simulation prédit un basculement des fibres.

Jusqu'à présent les simulations ont été réalisées avec un coefficient de diffusion constant. Une comparaison a été réalisée entre un calcul avec un coefficient de diffusion constant $\left(C_{I}=0,02\right)$ et un calcul avec un coefficient de diffusion variant avec la distance moyenne entre fibres (Éq. (10)). La longueur des fibres et leur diamètre ont été choisis respectivement à $280 \mu \mathrm{m}$ et $14 \mu \mathrm{m}$. Pour la figure 4 le coefficient $C_{I}^{*}$ a été choisi de telle manière que les viscosités au niveau du plateau soient égales pour $C_{I}^{*}=0,00086$. La différence entre les deux méthodes n'est pas très importante (Fig. 3).

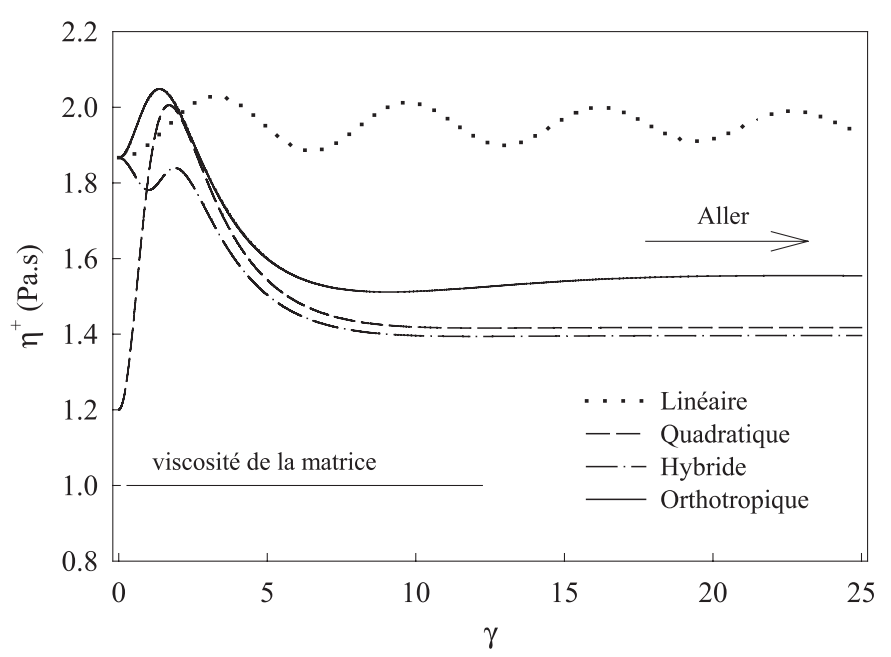

(a)

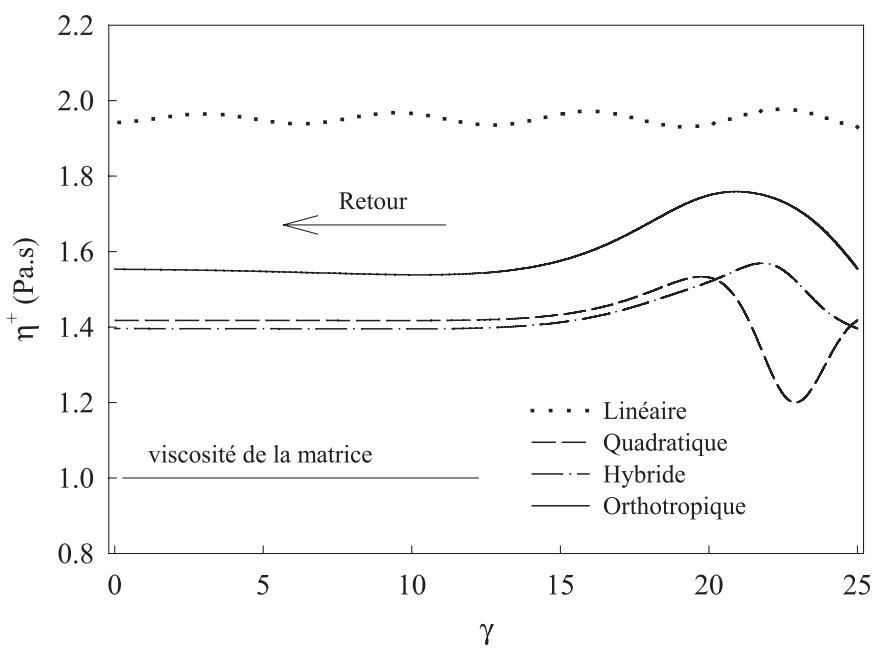

(b)

Fig. 1. Variation de la viscosité en fonction de la déformation sous cisaillement simple dans un écoulement aller (a) et retour (b) avec différentes approximations de fermeture.

Dans un fluide newtonien purement visqueux, les différences de contraintes normales sont nulles. La présence des fibres induira l'apparition de différences de contraintes normales. À partir de l'équation (15) la première et la seconde différence de contraintes normales sont respectivement définies par :

$$
\begin{aligned}
& N_{1}=\sigma_{11}-\sigma_{22}=2 \phi \eta_{0} \mu_{2} \dot{\gamma}\left(a_{1211}-a_{1222}\right) \\
& N_{2}=\sigma_{22}-\sigma_{33}=2 \phi \eta_{0} \mu_{2} \dot{\gamma}\left(a_{1222}-a_{1233}\right)
\end{aligned}
$$

L'évolution de ces deux grandeurs a été calculée en fonction de la déformation en utilisant le coefficient de diffusion variable (Fig. 4). Au démarrage elles présentent des pics puis elles se stabilisent. On constate que l'intensité de $N_{1}$ est beaucoup plus grande que celle de $N_{2}$. Lorsque le sens de l'écoulement est inversé, les grandeurs changent de signe, ensuite elles redeviennent positives et des pics de plus faibles intensités sont à nouveau observés (Fig. 4). 


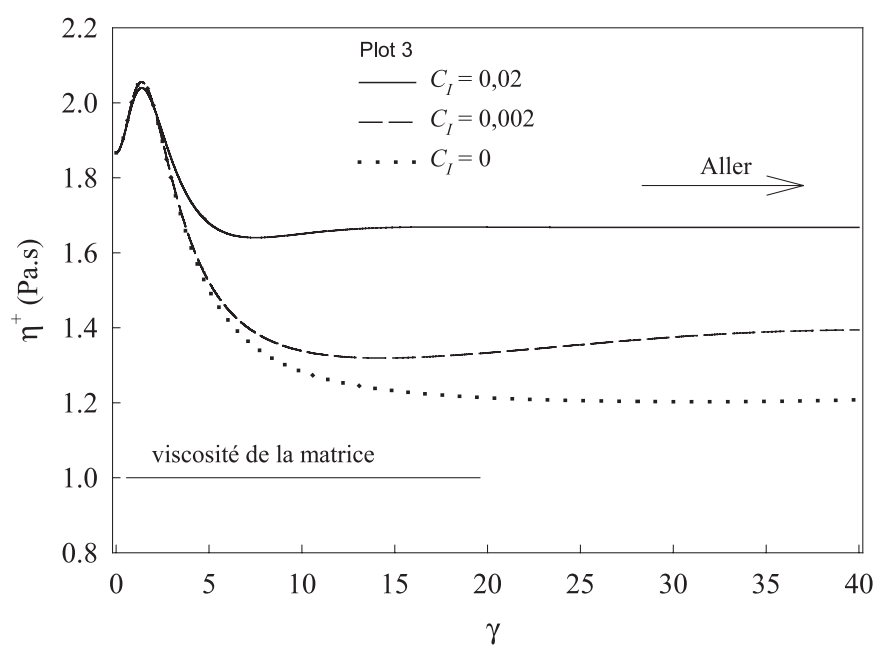

(a)

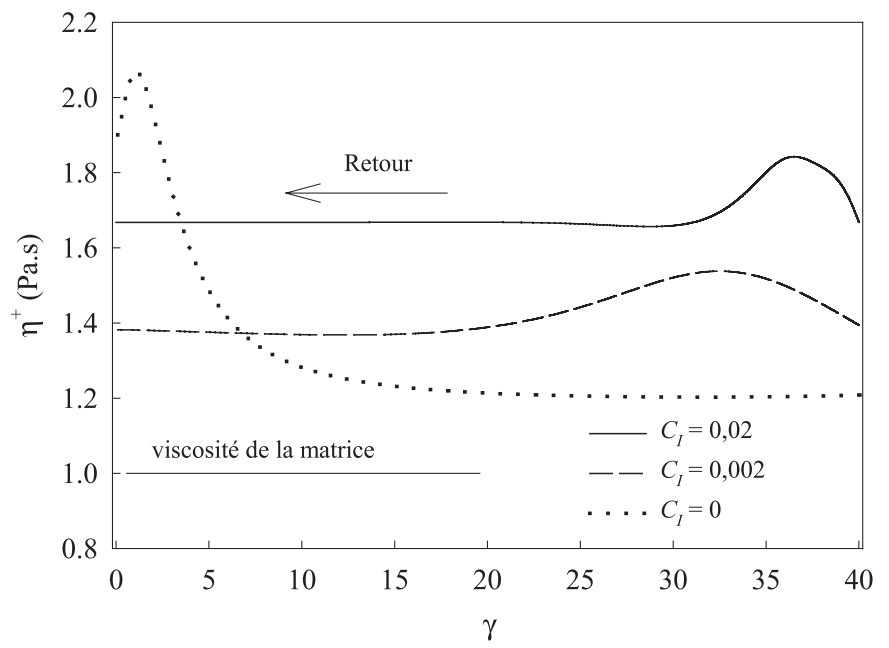

(b)

Fig. 2. Variation de la viscosité en fonction de la déformation sous cisaillement simple dans un écoulement aller (a) et retour (b) pour différentes valeurs du coefficient $C_{I}$.

\section{Expériences et confrontation}

Un polypropylène non-chargé (PP0, Targor PPN 0160D1) et un polypropylène chargé à $30 \%$ en poids de fibres de verre courtes (PP30, Targor Hostacom G3 N01L) ont été utilisés. Dans le composite utilisé le rapport de forme varie à l'intérieur d'une plage qui est importante. Cette distribution de rapport de forme a été mesurée et la valeur moyenne est environ égale à 20. Dans le modèle, on suppose que le rapport de forme est le même pour toute la population de fibres. Les caractéristiques des matériaux utilisés sont rassemblées dans le tableau 2.

Le matériau est d'abord mélangé dans un mélangeur interne (Brabender) à une vitesse de rotation contrôlée de $40 \mathrm{rpm}$ à $200{ }^{\circ} \mathrm{C}$ sous une atmosphère d'azote pendant 4 minutes. Ensuite il est moulé par compression à $200{ }^{\circ} \mathrm{C}$ pour obtenir des pastilles. Tous les échantillons sont préparés de la même manière. On suppose que

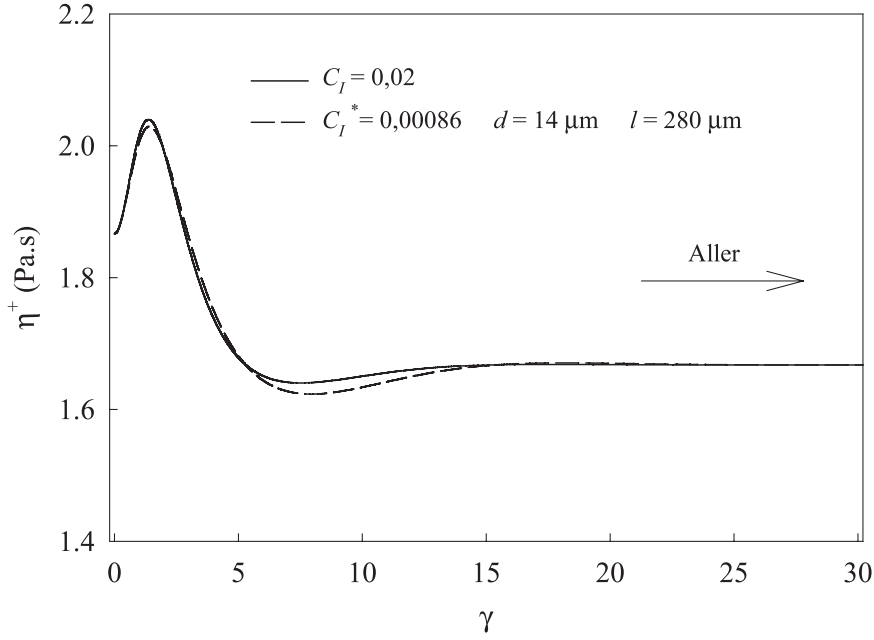

(a)

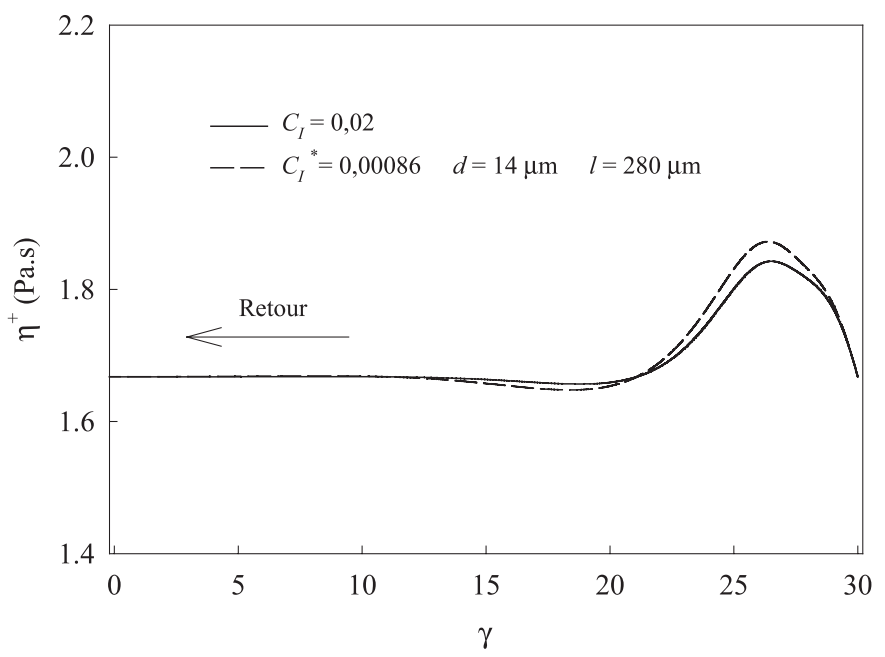

(b)

Fig. 3. Variation de la viscosité en fonction de la déformation sous cisaillement simple dans un écoulement aller (a) et retour (b) pour un coefficient de diffusion constant et pour un coefficient de diffusion dépendant de la distance entre fibres.

l'orientation des fibres dans les échantillons est isotrope. Ceci a déjà été vérifié dans des travaux précédents [19].

Les expériences ont été réalisées avec des rhéomètres à contraintes imposées, le SR 5000 (Rheometric Scientific) et le CSM (Bohlin). Une géométrie plan-plan est utilisée, la température est $200{ }^{\circ} \mathrm{C}$ et les expériences ont été effectuées sous azote. Les plateaux ont un rayon $R$ de $12,5 \mathrm{~mm}$ et l'entrefer est compris entre 1,1 et 1,9 mm.

Il est essentiel de vérifier la stabilité thermique des matériaux avant toute autre étude. Des expériences ont été effectuées sur le polypropylène sans fibre, et un taux de $1 \%$ d'un stabilisant approprié, l'Irganox B225 (Ciba Specialty Chemical Inc.), est nécessaire pour prévenir la dégradation thermique du PP. En ajoutant le stabilisant, la réduction de la viscosité est de $0,4 \%$ par heure, elle se stabilise à $4,3 \%$ et la viscosité reste constante pour une période de 17 heures. La stabilité du PP et des PP chargés 


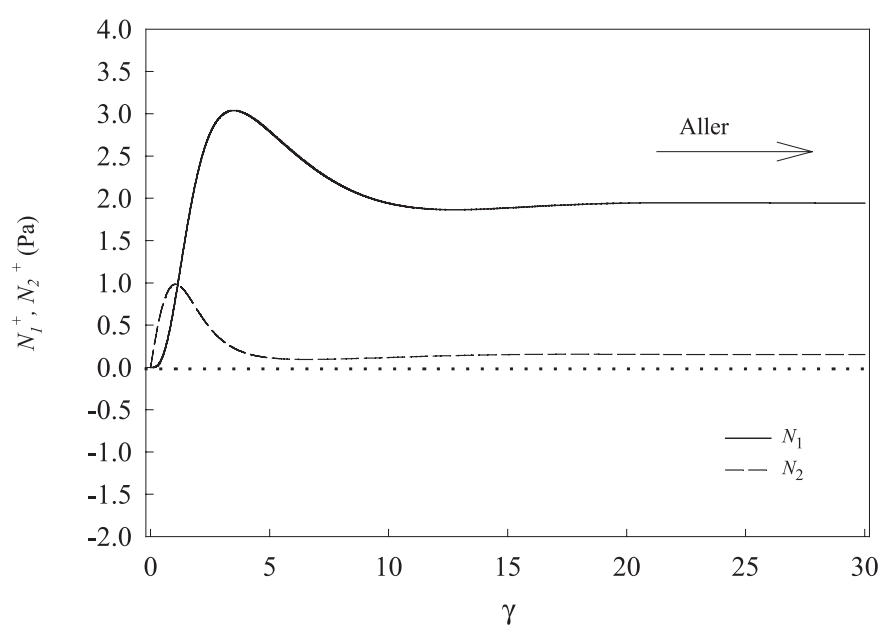

(a)

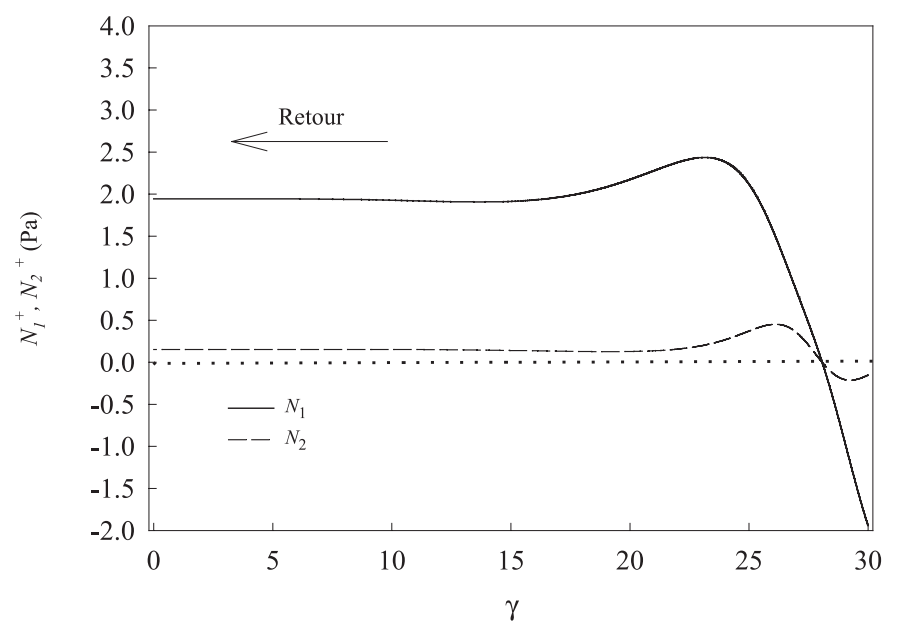

(b)

Fig. 4. Variation de la première et de la seconde différences de contraintes normales en fonction de la déformation sous cisaillement simple dans un écoulement aller (a) et retour (b) avec un coefficient de diffusion dépendant de la distance entre fibres.

Tableau 2. Propriétés du composite PP30.

\begin{tabular}{lc}
\hline Densité du polymère fondu à $200{ }^{\circ} \mathrm{C}$ & $0,76 \mathrm{~g} \cdot \mathrm{cm}^{-3}$ \\
Densité des fibres & $2,5 \mathrm{~g} \cdot \mathrm{cm}^{-3}$ \\
Fraction massique des fibres & 0,3 \\
Fraction volumique des fibres & $\phi=0,115$ \\
Longueur moyenne des fibres & $l=280 \mu \mathrm{m}$ \\
Diamètre des fibres & $d=14 \mu \mathrm{m}$ \\
Rapport de forme moyen des fibres & $r \approx 20$ \\
\hline
\end{tabular}

de fibres devient acceptable pour qu'on puisse continuer les études sur ces matériaux.

Il est également primordial de vérifier que les propriétés rhéologiques ne dépendent pas de l'entrefer entre les deux plateaux. Les essais réalisés avec différentes valeurs d'entrefers ont donné les mêmes résultats et ont permis de vérifier l'absence de glissement à la paroi.

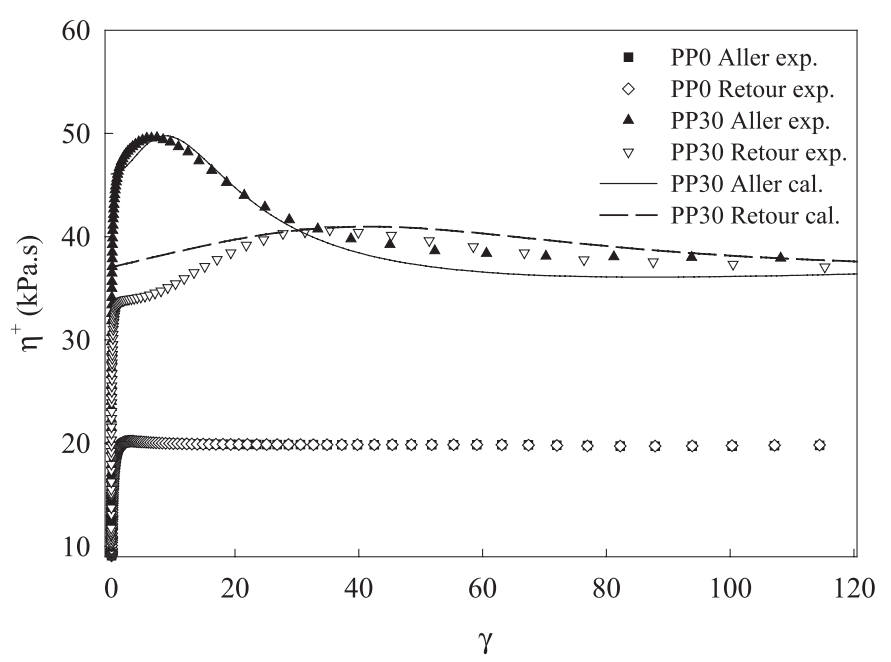

Fig. 5. Évolution de la viscosité du PP0 et du PP30 dans un écoulement aller et un écoulement retour sous cisaillement simple.

L'angle $\theta$ décrit l'angle de rotation du plateau supérieur par rapport à sa position de départ. La déformation représente les changements relatifs dans les dimensions et les angles de l'élément de volume dans une position donnée. Ici, la déformation de l'échantillon a été définie par $\gamma=\theta R / H$.

La figure 5 montre les données en fluage (en géométrie plan-plan) pour le polypropylène non-chargé et le polypropylène chargé de $30 \%$ en masse de fibres de verre à une contrainte imposée de $1000 \mathrm{~Pa}$. On remarque que la viscosité transitoire du PP0 ne montre aucun pic, tandis qu'un grand pic de viscosité est constaté pour le premier essai positif, l'« aller », sur le PP30. On remarque que la viscosité atteint le maximum à une déformation de presque 10 . Dans un essai consécutif négatif, le « retour », une vitesse de déformation négative a été immédiatement appliquée après l'essai positif. On note un pic de viscosité avec une plus petite amplitude à une déformation de presque 40 . Ce pic de retour est dû au basculement de la plupart des fibres dans le sens opposé. Au départ les fibres ont une orientation aléatoire, et sous un écoulement en cisaillement elles s'orientent dans la direction de l'écoulement. Le pic de viscosité est dû à la rotation et l'orientation privilégiée des fibres dans le plan de cisaillement. La plupart des fibres passent par un angle de $45^{\circ}$ au sommet de ce pic (Laun [20]) pour s'orienter dans le plan de cisaillement en arrivant au palier du régime permanent. Même si les fibres sont orientées en arrivant au plateau du régime établi de l'essai positif, mais elles ne sont pas totalement alignées dans le sens de l'écoulement. En changeant le sens de l'écoulement, les fibres basculent d'un angle inférieur à $180^{\circ}$ et s'orientent à nouveau dans le sens de l'écoulement.

Des résultats et la comparaison des prédictions du modèle pour des expériences de démarrage (stress growth) sont donnés dans [21].

Le modèle a été utilisé pour simuler l'écoulement du composite dans le rhéomètre. En supposant que l'état d'orientation est uniforme dans l'épaisseur à un rayon 
donné, le gradient de vitesse est alors uniforme dans l'épaisseur. Pour tout rayon, l'évolution de l'orientation au cours du temps et donc au cours de la déformation peut être alors évaluée à l'aide de l'équation (6). Connaissant l'orientation, la contrainte de cisaillement peut être déterminée à l'aide de l'équation (15) en considérant que la vitesse de cisaillement, $\dot{\gamma}$, est constante, et supposant que la viscosité de la matrice est constante. Finalement, le couple calculé exercé sur l'un ou l'autre des plateaux peut s'écrire sous la forme suivante :

$$
C_{\gamma}=\int_{0}^{R} 2 \pi r^{2} \sigma_{\theta z}(r) \mathrm{d} r
$$

Connaissant ce couple calculé en fonction de la déformation, la viscosité calculée s'écrit :

$$
\eta_{\gamma}=\frac{2 C_{\gamma} H}{\pi \omega R^{4}}
$$

où $\omega$ est la vitesse de rotation du plateau supérieur.

Par comparaison des viscosités calculées et expérimentales, il a été possible de déterminer des valeurs du coefficient $C_{I}^{*}$ permettant d'obtenir les mêmes allures de courbes. Cependant il a été constaté que les largeurs des pics mesurées et calculées sont très différentes : typiquement 10 pour le calcul et 50 pour les expériences sur le pic aller avec le PP30. Il faut rappeler que les modèles présentés et utilisés jusque là, ont été établis pour des suspensions diluées ou semi-diluées de fibres. Les composites utilisés sont des composites industriels contenant des grandes quantités de fibres correspondant à des solutions concentrées. Le modèle de Jeffery [1] a été établi pour une fibre isolée et donc sans interaction entre fibres ce qui est la réalité pour les solutions diluées. Ce modèle donne un mouvement de fibres qui est proche de la déformation affine de la matrice. Le terme de Folgar et Tucker [8] est un terme de diffusion qui ajoute un mouvement brownien aux rotations de la fibre. Il simule les contacts entre fibres pour des solutions semi-diluées de fibres. Il ne modifie pas les vitesses de rotation de la fibre, mais simplement ajoute un « bruit ». Dans le cas des solutions concentrées, apparaissent des phénomènes d'enchevêtrement et d'agrégation, et le mouvement des fibres est éloigné de la déformation affine de la matrice. Ce décalage entre le mouvement ralenti de la particule et le mouvement de la matrice peut être introduit sous la forme d'un glissement. Dans l'écoulement de systèmes complexes la notion de glissement a déjà été introduite. Dans un mélange de polymère Lacroix et al. [22] introduisent un glissement entre le mouvement de la phase dispersée et la matrice. Phan-Thien et al. [23,24] étudient la rhéologie d'une suspension de sphères. Ils supposent qu'elles s'agrègent par paires. Ces paires s'orientent sous l'effet des déformations de la matrice. Un tenseur des gradients de vitesses effectives est utilisé avec un facteur de glissement. Dans ce travail où la concentration de fibres est importante, le mouvement de rotation des fibres est beaucoup plus lent que prévu par le modèle de Jeffery. Les fibres ont de nombreux contacts et interactions entre elles et sont ralenties dans leur mouvement. Il est alors
Tableau 3. Coefficients du modèle pour le composite PP30.

\begin{tabular}{lc}
\hline Coefficient de couplage & $\mu_{2}=55$ \\
Coefficient d'interaction & $C_{I}^{*}=0,00018$ \\
Coefficient de glissement & $\alpha=0,19$ \\
\hline
\end{tabular}

introduit une déformation non-affine sous cisaillement simple dans la déformation calculée $\gamma_{\text {cal }}$ en ajoutant un coefficient de glissement $\alpha$ sous la forme suivante :

$$
\gamma_{\text {cal }}=\alpha \dot{\gamma} t
$$

La viscosité calculée a été tracée en fonction de la déformation calculée. La comparaison avec les expériences s'est avérée à ce moment là, intéressante lorsque les paramètres du modèle sont choisis correctement (Fig. 5). Il y a maintenant trois paramètres dans le modèle : le coefficient de couplage $\mu_{2}$, le coefficient de diffusion $C_{I}^{*}$, et le coefficient de glissement $\alpha$. Dans la figure 5 ont été comparé la viscosité relative du PP30 en fonction de la déformation dans un écoulement aller et retour obtenue expérimentalement et calculée avec pour les 3 coefficients les valeurs suivantes : $\mu_{2}=55 ; C_{I}^{*}=0,00018$ et $\alpha=0,19$. Les valeurs des coefficients sont rassemblées dans le tableau 3. La valeur de $\mu_{2}$ peut être comparée à la valeur théorique obtenue à l'aide de l'équation (16) et pour un rapport de forme de 20 on obtient $\mu_{2}=133$.

Dans cette étude le coefficient de diffusion dépend de la distance moyenne entre fibres qui dépend lui-même de l'état d'orientation des fibres. Pour le PP30 la distance moyenne entre fibres varie de $4,8 \mu \mathrm{m}$ pour un état d'orientation isotrope à $36,6 \mu \mathrm{m}$ pour un état d'orientation parfaitement aligné (Éq. (10)). Dans la simulation le coefficient de diffusion évolue entre 0,0146 et 0,0019 (Éq. (9)). Ces valeurs peuvent être comparées aux valeurs obtenues pour le PP30 à l'aide de l'équation de Bay [9] (Éq. (7)) qui donne $C_{I}=0,0036$ et de l'équation de Phan-Thien et al. [11] (Éq. (8)) qui donne $C_{I}=0,012$. Il est intéressant de constater que les valeurs obtenues pour le coefficient $C_{I}$ dans cette étude évoluent autour de celles obtenu par l'équation de Bay [9]. Elles ont pourtant été obtenues de deux manières très différentes. Bay a établit son modèle empirique (Éq. (7)) en effectuant des mesures d'orientation de fibres sur des pièces injectées. Dans cette étude, le coefficient de diffusion $C_{I}$ a été déterminé à partir d'essais de caractérisation rhéologique sous cisaillement simple dans un écoulement aller et retour.

\section{Simulation et amélioration des procédés}

Ces modèles permettent de comprendre et de prédire le comportement des composites polymères chargés de fibres courtes. Ils sont également utilisés dans des calculs simulant l'écoulement dans des géométries simples ou dans des procédés de mise en forme. Ausias et al. [25] et Chiba et al. [26] simulent l'écoulement d'un composite dans une géométrie simple. Le calcul permet 
de bien décrire les effets du couplage entre orientation et écoulement : le mouvement du fluide déplace et fait tourner les fibres, la présence de celles-ci et leur état d'orientation modifient le profil de vitesse. Poitou et al. [27] réalisent cette simulation dans des écoulements tri-dimensionnels. Ces modèles sont également introduits dans des codes commerciaux de simulation de l'injection plastique comme MoldFlow.

Récemment, des comparaisons entre des simulations du procédé d'injection et des expériences ont été présentées par Tucker et Huynh [28]. Elles semblent montrer que dans des écoulements se situant juste après le point d'injection, les fibres tournent et s'orientent plus lentement que ne le prévoit la simulation. Ce résultat va dans le sens des résultats présentés ci-dessus et justifie l'introduction d'un coefficient de glissement. Ces outils permettent de comprendre et de prédire les phénomènes d'orientation des fibres dans les outillages de mise en forme et dans certains cas d'aider à optimiser les outillages. Ausias et al. [29] étudient la fabrication de tubes en composite. Grâce aux modèles rhéologiques et à la simulation numérique, ils proposent un nouveau type de filière et vérifient que celle-ci améliore effectivement l'état d'orientation dans des fibres dans le tube (Jarrin et al. [30]).

\section{Conclusion}

L'utilisation de fibres courtes en régimes concentrées dans les thermoplastiques industriels est courante. Cette étude permet de mieux comprendre le comportement rhéologique des composites polymères chargés de fibres et de proposer une modification des modèles existants sous cisaillement simple en introduisant un coefficient de glissement.

La présence de ces fibres provoque des comportements rhéologiques complexes. Des essais transitoires consécutifs ont été effectués dans deux directions différentes. Dans un premier essai positif, on constate un pic pour la viscosité. En arrêtant le premier essai et en le reprenant immédiatement dans le sens opposé, un pic de retour avec une plus petite amplitude réapparaît dans des déformations plus élevées. Le pic de viscosité est dû à l'orientation des fibres dans le sens de l'écoulement et le pic de retour est dû au basculement des fibres dans le sens inverse.

La confrontation du modèle basé sur les modèles de Folgar et Tucker [8] et de Lipscomb [16] avec les résultats expérimentaux ne montre pas une bonne corrélation. Ce qui peut être expliqué par des interactions entre les fibres dans des suspensions concentrées qui causent une déformation non-affine. Afin de modifier le modèle un coefficient de glissement est introduit dans le modèle de base mais il reste toujours beaucoup de travail pour mieux caractériser ces matériaux et établir des modèles pour des solutions concentrées de fibres.
Remerciements. Ce travail a été supporté par le programme de collaboration France-Québec et financé par le NSERC (Natural Science and Engineering Research council of Canada). Les matériaux utilisés dans cette étude ont été fournis par Targor et les auteurs tiennent à remercier le Dr. G. Krotkine. Ils remercient également le Pr. C.L. Tucker pour son aide dans l'utilisation des calculs d'approximations de fermeture développées dans son équipe.

\section{Références}

[1] G.B. Jeffery, The motion of ellipsoidal particles immersed in a viscous fluid, Proc. Roy. Soc. A102 (1922) 161-179

[2] S.G. Advani, C.L. Tucker, Closure approximations for three-dimensional structure tensors, J. Rheol. 34 (1990) 367-386

[3] J.S. Cintra, C.L. Tucker, Orthotropic closure approximations for flow-induced fiber orientation, J. Rheol. 39(6) (1995) 1095-1122

[4] E.D. Wetzel, C.L. Tucker, Area tensors for modeling microstructure during laminar liquid-liquid mixing, Int. J. of Multiphase Flow 25 (1999) 35-61

[5] V. Verleye, F. Dupret, Numerical prediction of fiber orientation in complex injection molded parts, Proceedings of the ASME Winter Annual Meeting, MD, vol. 49. HTDvol. 283 (1994) 264-279

[6] B.E. VerWeyst, Numerical Prediction of Flow-Induced Fiber Orientation in 3D Geometries, Ph.D. Thesis, University of Illinois at Urbana-Champaign, Urbana, IL, 1998

[7] E.D. Wetzel, C.L. Tucker, Area tensors for modeling microstructure during laminar liquid-liquid mixing, Int. J. of Multiphase Flow 25 (1999) 35-61

[8] F. Folgar, C.L. Tucker, Orientation behavior of fibers in concentrated suspensions, J. Reinforced Plastics and Composites 3 (1984) 99-119

[9] R.S. Bay, Fiber orientation in injection molded composites : A comparison of theory and experiment, Ph.D. dissertation, University of Illinois, Urbana-Champaign, 1991

[10] X.-J. Fan, N. Phan-Thien, R. Zheng, A direct simulation of fibre suspensions, J. Non-Newtonian Fluid Mech. 74 (1998) 113-135

[11] N. Phan-Thien, X.-J. Fan, R.I. Tanner, R. Zheng, FolgarTucker constant for a fibre suspension in a Newtonian Fluid, J. Non-Newtonian Fluid Mech. 103 (2002) 251-260

[12] S. Ranganathan, S.G. Advani, Fiber-fiber interactions in homogeneous flows of nondilute suspensions, J. Rheol. 35(8) (1991) 1499-1522

[13] A. Aït-Kadi, M. Grmela, Modeling the rheological behavior of fiber suspensions in viscoelastic media, J. NonNewtonian Fluid Mech. 53 (1994) 65-81

[14] G.K. Batchelor, The stress system in a suspension of freeforce particles, J. Fluid Mech. 41 (1970) 545-570

[15] J.L. Ericksen, Transversely Isotropic fluid, Kolloid Z. 173 (1960) 117-122

[16] G.G. Lipscomb, Analysis of suspension rheology in complex flows, Ph.D. dissertation, University of California, Berkeley, 1987

[17] G.L. Hand, A theory of anisotropic fluids, J. Fluid Mech. 13 (1962) 33-46 
[18] H. Giesekus, Elasto-viskose flüssigkeiten, für die in stationären schichtströmungen sämtliche normalspannungskomponenten verschieden grob sind, Rheol. Acta. 2(50) (1962) 50-62

[19] G. Ausias, J.F. Agassant, M. Vincent, P.G. Lafleur, P.A. Lavoie, P.J. Carreau, Rheology of Short Fiber Reinforced Polypropylene, J. Rheol. 36 (1992) 525-542

[20] H.M. Laun, Orientation effects and rheology of short glass fiber-reinforced thermoplastics, Coll. Polym. Sci. 262 (1984) 257-269

[21] M. Sepehr, G. Ausias, P.J. Carreau, Short fiber filled polypropylene rheology under transient shear flows, soumis au J. of Non-Newtonian Fluid Mech. (2003)

[22] C. Lacroix, M. Grmela, P.J. Carreau, Morphological evolution of immiscible polymer blends in simple shear and elongational flows, J. Non-Newtonian Fluid Mech. 86 (1999) 37-59

[23] N. Phan-Thien, X.J. Fan, B.C. Khoo, A new constitutive model for monodispersed suspensions of spheres at high concentrations, Rheol. Acta. 38 (1999) 297-304

[24] N. Phan-Thien, X.J. Fan, R. Zheng, A numerical simulation of suspension flow using a constitutive model based on anisotropic interparticle interactions, Rheol. Acta. 39 (2000) 122-130
[25] G. Ausias, J.F. Agassant, M. Vincent, Flow and Fibre Orientation Calculations in Reinforced Thermoplastic Extruded Tubes, International Polymer Processing, IX (1) (1994) 51-59

[26] K. Chiba, K. Yasuda, K. Nakamura, Numerical solution of fiber suspension flow through a parallel plate channel by coupling flow field with fiber orientation distribution, J. Non-Newtonian Fluid Mech. 99 (2001) 145-157

[27] A. Poitou, F. Chinesta, R. Torres, Numerical simulation of the steady recirculating flow of fiber suspensions, J. Non-Newtonian Fluid Mech. 90 (2000) 65-80

[28] C.L. Tucker, H.M. Huynh, Fiber orientation in short flow length parts: limitation of current predictions, 17th Annual Meeting of the PPS, Montreal May, 2001, pp. $21-24$

[29] G. Ausias, J.F. Agassant, M. Vincent, Optimization of the tube extrusion die for short fiber filled polymers, Composites Sci. and Technology 56 (1996) 719-724

[30] J. Jarrin, G. Ausias, M. Vincent, E. Vinciguerra, F. Dawans, Production of tubes of thermoplastic material reinforced by fibres of controlled orientation and equipment for preparation, Brevet européen $\mathrm{n}^{\circ}$ EP 0503364, Brevet USA $n^{\circ}$ US 5307843, 1992, Accesion number: 199703-E2-D-0039. 